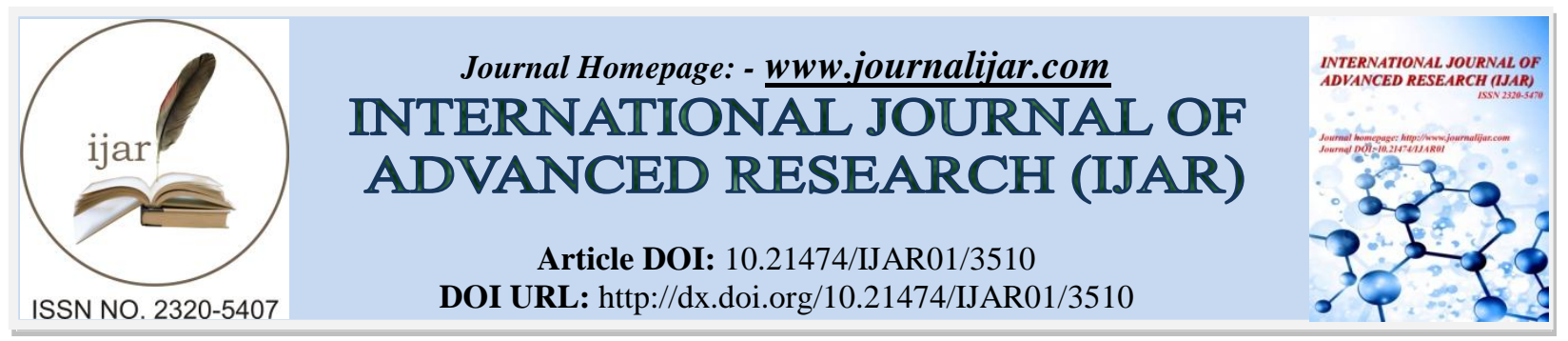

RESEARCH ARTICLE

\title{
DENGUE FEVER IN CHILDREN: ROLE OF ULTRASONOGRAPHY.
}

"Dr. Sujit Mulay ${ }^{1}$, Dr. C. S. Rajput ${ }^{2}$, Dr. Sudha Bhave ${ }^{3}$ and Dr. Suresh Waydande ${ }^{4}$.

1. Junior Resident, Department of pediatrics, Bharati vidyappeth university medical College and Hospital, Sangli.

2. Professor, Department of pediatrics, Bharati vidyappeth university medical College and Hospital, Sangli.

3. Associate Professor, Department of pediatrics, Bharati vidyappeth university medical College and Hospital, Sangli.

4. Assistant Professor Department of pediatrics, Bharati vidyappeth university medical College and Hospital, Sangli.

\section{Manuscript Info}

Manuscript History

Received: 11 January 2017

Final Accepted: 13 February 2017

Published: March 2017

Key words:-

Dengue Fever, Gall bladder thickening,

Hepatomegaly, Splenomegaly,

Ultrasonography

\section{Abstract}

Introduction: Dengue fever is one of the most important emerging vector-borne viral diseases. There are four serotypes of dengue viruses, each of which is capable of causing self-limited dengue fever or even life-threatening dengue haemorrhagic fever and dengue shock syndrome. The aim of this study was to evaluate the role of sonographic findings as early diagnostic modality in children with dengue fever.

Materials \& Methods: This cross-sectional observational study was carried out in the department of pediatrics of a tertiary care hospital. A total of 88 patients who were clinically and serologically (NS1 antigen/ $\mathrm{IgM} / \mathrm{IgG}$ antibody) diagnosed as dengue positive were included in this study.These patients also underwent USG abdomen and chest within the first week of the illness.

Results: Out of the 88 confirmed dengue cases,56 (63.6\%) cases were males. All the cases had fever $(100 \%)$. Other clinical symptoms were vomiting in $64.8 \%$, abdominal pain in $62.5 \%$, bodyache $53.4 \%$, Shock in $28.4 \%$, headache in $48.9 \%$ and rashes in $23.9 \%$.The USG findings were hepatomegaly (39.8\%), gall bladder thickening (53.4\%), splenomegaly (20.5\%), pleural effusion (38.6\%) and ascites $(30.7 \%)$.Overall $65(73.8 \%)$ out of 88 cases had one of the positive finding in ultrasonography.

Conclusion: Ultrasonography can be used as a first-line imaging modality in all patients with suspected dengue fever to detect early signs suggestive of the disease.

Copy Right, IJAR, 2017,. All rights reserved.

\section{Introduction:-}

Dengue fever is an acute febrile infectious disease in subtropical and tropical areas that is progressively making its way from being "one of the great neglected diseases of mankind" [1] towards being acknowledged as one of the world's major infectious diseases [2]. In the last 50 years, incidence of dengue has increased 30-fold with increasing geographic expansion to new countries and from urban to rural settings. An estimated 50 million dengue infections occur annually and approximately 2.5 billion people live in dengue endemic countries [3]. 
The etiologic agents include all four serotypes which belong to the genus Flavivirus in the family Flaviviridae. The principle vector is mosquito, AedesAegypti, which breeds largely indoors in clean waters mainly in artificial water containers, and feeds on humans in daytime [4].

The clinical manifestations of dengue vary with the age and immunity of the patient. It can present as 1) In apparent infection 2) non- specific febrile illness, 3) classic dengue fever, 4) dengue hemorrhagic fever, 5) Dengue shock syndrome (DSS) and 6) encephalopathy and fulminant liver failure [5,6].

Guzman et al. studied to show that most cases present as classic dengue fever (DF) with high fever, retro-orbital pain, severe myalgia/arthralgia, and rash. However, in some cases, illness progresses to life-threatening dengue hemorrhagic fever/dengue shock syndrome (DHF/DSS), characterized by vascular leakage leading to hypovolemic shock and a case fatality rate up to $5 \%[7,8]$.

Hammond et al. [9] studied to show that preschool children and infants have rather more often nonspecific febrile illnesses while preadolescent children often develop fever and moreover younger children with DHF are known to experience more severe clinical outcome (e.g. higher case fatality ratio) than adults.

Early identification of patients at risk of developing severe dengue is critical to provide timely supportive care, which can reduce the risk of mortality to $<1 \%$ [10]. Distinguishing dengue from other febrile illnesses (OFIs) early in illness is challenging, since symptoms are non-specific and common to other febrile illnesses such as malaria, leptospirosis, rickettsiosis, and typhoid fever in dengue-endemic countries [11].

Positive serology (anti dengue antibody) is the mainstay in the diagnosis of DF. But serology takes approximately 7 days to give a positive result [12,13]. The diagnosis of DF is often delayed owing to time taken for availability of serology test results [14]. Ultrasonography (USG) is a cheap, rapid and widely available non-invasive imaging method $[15,16]$. Some authors concluded findings of hepatomegaly, pericholecystic edema, thickened gall bladder, ascites, pleural effusion (right sided/ both) and splenomegaly are early and significant markers of dengue, which can be confirmed by serology later on $[17,18]$. Several studies concluded that ultrasonography of the chest and abdomen can be an important adjunct to clinical profile in diagnosis DF and diagnosis can be made early in the course of disease compared with other modes of diagnosis [14]. It can be used as a first-line imaging modality in patients with suspected dengue fever to detect early signs suggestive of the disease prior to obtaining serologic confirmation test results, especially in a dengue fever epidemic area [17].

These findings may also occur in other viral infections and enteric fever but in other viral infections the historical profile, symptom complex evolution and physical findings do not mimic those of dengue fever [14]. Thus, the aim of present study was to evaluate the sonographic findings of dengue fever which may be useful as an early diagnostic tool.

\section{Materials and Methods:-}

Study Type \& Area:-

A prospective observational study was conducted at Department of Paediatrics of a tertiary care centre during July 2014 to August 2016.

\section{Inclusion Criteria:-}

1. Patient between the age group of $0-18$ years.

2. Patients with confirmed diagnosis of dengue fever (positive test for NS1 antigen/IgM dengue/IgG dengue).

\section{Exclusion Criteria:-}

1. Patient with serological test negative for dengue.

2. Patients having fever of more than 2 weeks duration.

\section{Sample Size:-}

Consecutive type of non-probability sampling was used for the selection of study subjects. A total of 88 diagnosed pediatric patients of Dengue fever admitted in our hospital were taken for study after informed consent from parents. 


\section{Study Methodology:-}

All patients were admitted and treated as indoor patients. On admission, detailed history and complete physical examination findings were recorded. The vitals (temperature, pulse, respiration, blood pressure) of the patients were recorded on admission and thereafter till discharge.In all patients, complete blood count and serological tests for dengue (NS1 antigen/ IgM dengue/ IgG dengue) were carried out depending on duration of fever on presentation.

\section{Following laboratory investigations were done in all patients on admission:-}

1. Dengue NS1 antigen test/ IgM dengue antibody/ IgG dengue antibody- depending on duration of fever on presentation. All the three tests will be done by the ELISA (enzyme linked immunosorbant assay) technique.

2. Complete blood count (CBC) including platelet count.

3. Coagulation Profile

4. USG abdomen

Other investigations like liver function test, renal function test, S. electrolyte, X-ray chest/abdomen, blood culture, peripheral smear for malarial parasite were performed based on the clinical presentation.

Based on the investigation results, patients were classified into 3 categories as per WHO guidelines: Dengue fever, Dengue Hemorrhagic fever and Dengue shock syndrome. All patients will be treated as per WHO guidelinesDengue guidelines for diagnosis, treatment, prevention and control [19].

\section{Results:-}

Out of the 88 confirmed dengue cases,56(63.6\%) were males while $32(36.4 \%)$ were females. Fever was present in all the cases, while other features were: vomiting (64.8\%), abdominal pain (62.5\%), bodyache $(53.4 \%)$, shock $(28.4 \%)$, headache $(48.9 \%)$ and rashes $(23.9 \%)$ (Table 1). Investigations revealed thrombocytopenia in $78.4 \%$, hemoconcentration in $48.9 \%$, raised liver enzymes in $68.2 \%$ while raised creatinine levels in $2.3 \%$ patients (Table 2).The ultrasonographic findings showed hepatomegaly in 35(39.8\%) cases, gall bladder thickening in $47(53.4 \%)$ cases, splenomegaly in $18(20.5 \%)$ cases, pleural effusion in 34(38.6\%) cases while ascites was seen in $27(30.7 \%)$ cases (Table 3).Overall $65(73.8 \%)$ out of 88 cases had one of the positive finding in ultrasonography. Final Diagnosis of Dengue was given in $15.9 \%$ cases while diagnosis of dengue with warning signs and severe dengue was given in $55.7 \%$ and $28.4 \%$ cases respectively (Table 4). Out of total 88 patients, $2(2.27 \%)$ patients expired who were suffering from of severe dengue.

Table 1:- Distribution of patients according to presenting complaints.

\begin{tabular}{|c|c|c|}
\hline Presenting Complaints & $\mathbf{N}$ & \% \\
\hline Fever & 88 & $100 \%$ \\
\hline Headache & 43 & $48.9 \%$ \\
\hline Bodyache & 47 & $53.4 \%$ \\
\hline Rash & 21 & $23.9 \%$ \\
\hline Vomiting & 57 & $64.8 \%$ \\
\hline Abdominal Pain & 55 & $62.5 \%$ \\
\hline Shock & 25 & $28.4 \%$ \\
\hline
\end{tabular}

Table 2:- Distribution of patients according to laboratory findings.

\begin{tabular}{|c|c|c|}
\hline Lab Findings & N & \% \\
\hline Thrombocytopenia & 69 & $78.4 \%$ \\
\hline Hemoconcentration & 43 & $48.9 \%$ \\
\hline Raised Liver Enzymes & 60 & $68.2 \%$ \\
\hline S. Creatinine (> 1.2 mg\%) & 2 & $2.3 \%$ \\
\hline
\end{tabular}

Table 3:- Distribution of patients according to sonographic findings.

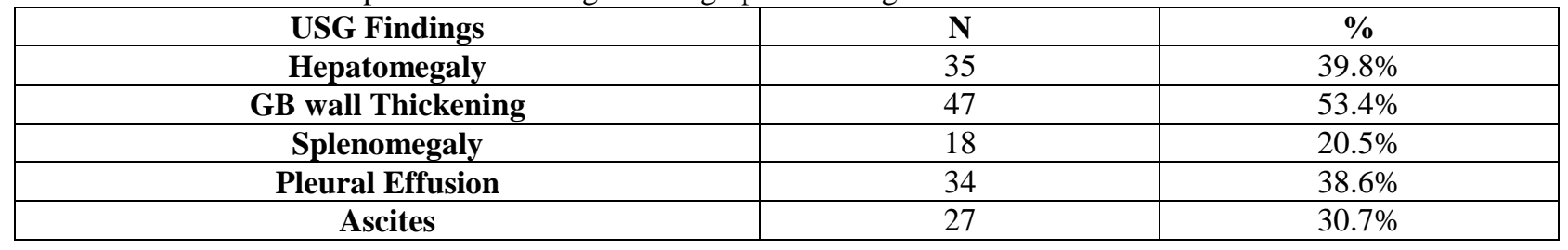


Table 4:- Distribution of patients as per Final Diagnosis.

\begin{tabular}{|c|c|c|}
\hline Diagnosis & $\mathbf{N}$ & $\%$ \\
\hline Dengue & 14 & $15.9 \%$ \\
\hline Dengue with warning signs & 49 & $55.7 \%$ \\
\hline Severe Dengue & 25 & $28.4 \%$ \\
\hline Total & 88 & $100.0 \%$ \\
\hline
\end{tabular}

\section{Discussion:-}

Dengue has become a major international public health concern in recent years [20]. It is emerging as one of the most important mosquito borne diseases in India. Aedes aegypti mosquitoes that transmit the disease breed in manmade containers such as tanks, pitchers, discarded containers etc. in which water has stagnated for over a week. Thus, the success of control measures have become a reflection of sanitation and hygienic practices achieved. The cases of dengue peak in the monsoon season in most parts of the country but have become perennial in the southern states and Gujarat [21,22].

Dengue has myriad clinical manifestations with unpredictable evolution and outcome. The disease typically begins with an acute febrile phase lasting 2-7 days and is accompanied by flushing, generalized body ache, myalgia, arthralgia and headache. Increased capillary permeability reflected by progressive increase in hematocrit heralds the beginning of critical phase at around 3-7 days of illness. Severe hemorrhagic manifestations and shock secondary to plasma leakage may occur at this stage. Leukopenia and declining platelet counts are also seen preceding this stage [23]. Serological diagnosis is confirmatory of dengue and includes direct methods such as virus isolation and NS1 antigen detection and indirect methods such as IgM and IgG antibody detection [24].As serological diagnosis is not available in most of the places in a resource scarce country like India, sonography has an increasingly important role. Sonography is a readily available and cost-effective method for the diagnosis, which impacts the management of patients of dengue. The aim of present study was thus to evaluate the role of sonographic findings as early diagnostic modality in children with dengue fever.

In present study, male predominance was seen with $63.6 \%$ male cases to $36.4 \%$ females with male to female ratio of 1.74:1. In a study by Hema $\mathrm{M}$ et al. [25], the sex ratio of male to female was 1.32: 1, while in that of Srinivasa et al. [26], it was 1.13:1. The sex distribution is also consistent with previous study findings that dengue fever occurs more in male sex [22,24,27].

In present study, fever was present in all the cases, while other features were: vomiting (64.8\%), abdominal pain $(62.5 \%)$, bodyache $(53.4 \%)$, shock $(28.4 \%)$, headache $(48.9 \%)$ and rashes $(23.9 \%)$. In the study by Srinivas et al. [26], fever and vomiting were the most frequent symptoms. Similar observation was also seen in earlier studies [14,28,29]. Srinivasa also observed rashes in 10.5\% cases while Ahmed FU et al [30] noted rashes in 12\% of children.

We also observe thrombocytopenia in $78.4 \%$ cases while hemoconcentration and raised liver enzymes were observed in $48.9 \%$ and $68.2 \%$ cases respectively. In the study by Mittal Hema et al. [25], thrombocytopenia and hepatic enzyme derangement was present in $92.6 \%$ and $60 \%$ of the cases respectively. In the study by Narayanan M. et al. [31], serum SGPT levels were elevated in $59.7 \%$ of the total patients in whom the test was performed. Serum SGPT levels were elevated in $65 \%$ of DF, $63.6 \%$ of DHF and $66 \%$ of DSS patients. In the study by Aggarwal Anju et al. [32], serum SGPT levels were elevated in $57 \%$ of the patients in whom the test was performed.

The ultrasonographic findings showed hepatomegaly in $35(39.8 \%)$ cases, gall bladder thickening in $47(53.4 \%)$ cases, splenomegaly in $18(20.5 \%)$ cases, pleural effusion in 34(38.6\%) cases while ascites was seen in $27(30.7 \%)$ cases. Overall $65(73.8 \%)$ out of 88 cases had one of the positive finding in ultrasonography. This clearly shows that Ultrasound can be used as an early, non-invasive and economical diagnostic tool.

In the study by srinivasa et al. [26], 72\% of the cases had ultrasound findings in the form of either hepatomegaly or gall bladder wall thickening. The ultrasound findings showed gall bladder wall thickening in $30.5 \%$. In a study by Quiroz-Moreno et al. [33] gallbladder thickening was seen in 86\% of the patients, Sai et al. [14] observed in 56\% patients whileThulkar et al. in $35.1 \%$ [34]. Setiwan et al. attempted to investigate whether gall bladder wall thickening measured by ultrasonography can be used to predict the onset of severe dengue fever. It is found that a 
thickness of $5 \mathrm{~mm}$ is useful as a criterion for identifying DHF patients at high risk of developing hypovolemic shock [35].

In our study pleural effusion or ascites was apparent on clinical examination in some of the cases. But sonography helped in diagnosing all of them. Similarly 15 cases of hepatomegaly and 11 cases of splenomegaly could not be diagnosed by clinical examination but were diagnosed correctly by ultrasonography. So this study clearly demonstrates the importance of ultrasonography in the accurate and complete clinical evaluation of dengue fever. All the cases which went into hypotension and shock showed gall bladder wall thickening, with moderate to severe pleural effusion and ascites.

\section{Conclusion:-}

Abdominal USG should be used as a first-line imaging modality in all patients with suspected dengue fever to detect early signs suggestive of the disease along with obtaining serologic confirmation tests.

\section{References:-}

1. Halstead, S. B.. The XXth century dengue pandemic: need for surveillance and research." World health statistics quarterly. Rapport trimestriel de statistiquessanitairesmondiales. 1992;45(2-3): 292-298.

2. Jelinek, T., N. Muhlberger, et al. Epidemiology and clinical features of imported dengue fever in Europe: sentinel surveillance data from TropNetEurop." Clinical infectious diseases: an official publication of the Infectious Diseases Society of America. 2002; 35(9): 1047-1052.

3. Nathan Michael B., Dr. DayalRenu-Drager, Guzman Maria."Epidemiology, Burden of disease and Transmission. WHO Dengue guidelines for diagnosis, treatment, prevention and control, New edition 2009.1: 3-17

4. Pancharoen C, Kulwichit W, Tantawichien T, Thisyakorn U, Thisyakorn C et al. "Dengue Infection: A Global Concern." J Med Assoc Thai. 2002;85 (Supp 1): S25 - S3

5. Innis BL. "Dengue and dengue haemorrhagic fever" In portar field JS, ed. Kass handbook of infectious disease; exotic virus infections, London Chapman and Hall Medical. 1995;103:41-46.

6. Isturiz RE, Gubler DJ, Brea del Castillo J. (2000) "Dengue and dengue hemorrhagic fever in Latin America and the Caribbean". Infect. Dis ClinNorth Am. 2000;14:121-40.

7. Sirivichayakul C. Dengue infection in children in Ratchaburi, Thailand. Clinical manifestations. PLoSNegl Trop Dis. 2012; 6(2):e1520.

8. Guzman MG, Halstead SB, Artsob H, Buchy P, Farrar J. Dengue: A continuing global threat. Nat Rev Microbiol. 2010; 8: S7-16.

9. Hammond SN, Balmaseda A, Perez L, Tellez Y, Saborio SI. Differences in dengue severity in infants, children, and adults in a 3-year hospitalbased study in Nicaragua. Am J Trop Med Hyg. 2005; 73: 1063-70.

10. Nieto NC, Khan K, Uhllah G, Teglas MB. The emergence and maintenance of vector-borne diseases in the khyberpakhtunkhwa province, and the federally administered tribal areas of pakistan. Front Physiol. 2012; $3: 250$.

11. Watt G, Jongsakul K, Chouriyagune C, Paris R. Differentiating dengue virus infection from scrub typhus in Thai adults with fever. Am J Trop Med Hyg. 2003;68:536-538.

12. Rahman M, Rahman K, Siddique AK, Shoma S, Kamal AH, Ali KS, et al. First outbreak of dengue haemorrhagic fever, Bangladesh. Emerg Infect Dis 2002; 8(7): 738-740.

13. Bhamarapravti N. Pathology of dengue haemorrhagic fever. In: Thongcharoen $P$ (ed) Denge/dengue haemorrhagic fever. WHO, Geneva 1993; pp.72-3.

14. Sai PMV, Dev B, Krishnan R. Role of ultrasound in dengue fever. British Journal of Radiology 2005, 78: 416418.

15. Venkata S, Dev B, Krishnan R. Role of ultrasound in dengue fever. Br J Radiol. 2005; 78: 416-418.

16. Thulkar S, Sharma S, Srivastava DN, Sharma SK, Berry M, Pandey RM et al. Sonographic findings in grade III dengue hemorrhagic fever in adults. J ClinUltrasound 2000; 28: 34-37.

17. Wu KL, Changchien CS, Kuo CH .Early abdominal sonographic findings in patients with dengue fever. Clin Ultrasound 2004; 32: 386-388.

18. Statler J, Mammen M, Lyons A, Sun W. Sonographic findings of healthy volunteers infected with dengue virus. J Clin Ultrasound 2008; 36: 413-417. 
19. Nathan Michael B., Dr. DayalRenu-Drager, Guzman Maria."Epidemiology, Burden of disease and Transmission. WHO Dengue guidelines for diagnosis, treatment, prevention and control, New edition 2009.1: 3-17

20. Dengue Haemoorrhagic fever: Diagnosis, treatment and controle, Geneva, World Health Organization, 1986.

21. National Vector Borne Disease Control programme. Annual Report 2014-15.

22. Santhosh VR, Patil PG, Srinath MG, Kumar A, Jain A, Archana M. Sonography in the diagnosis and assessment of dengue fever. J Clin Imaging Sci 2014;4:14.

23. Dengue: Guidelines for diagnosis, treatment, prevention and control. 2009. A joint publication of the World Health Organization (WHO) and the Special Programme for Research and Training in Tropical Diseases (TDR).

24. Martina BEE, Koraka P, Osterhaus ADME. Dengue virus pathogenesis: An integrated view. ClinMicrobiol Reviews. 2009; 10:564-81.

25. Mittal Hema et al. "Clinicohematological profile and platelet trends in children with dengue during 2010 epidemic in north India.” Indian J Pediatr., April 2012; volume 79, issue 4: 467-471.

26. Srinivasa S, Nawab T, Nair CC. Clinical profile and ultasonogaphic findings in children with dengue fever. Current Pediatrics. 2014;18(2).

27. Dhooria S. et al. "Clinical profile and outcome in children of dengue hemorrhagic fever in north India." Iran J Pediatr. Sep 2008; vol 18 (No.3): 222-228.

28. Srivastava VK, Suri S, Bhasin A, Srivastava L, Bharadwaj M. An epidemic of dengue hemorrhagic fever and dengue shock syndrome in Delhi: a clinical study. Ann TropPediatr 1990; 10: 329-334.

29. Bethell DB, Gamble J, Loc PP, Dung NM, Chau TTH, Loan HT, et al. Non-invasive measurement of microvascular leakage in patients with dengue hemorrhagic fever. ClinInfect Dis 2001; 32: 243-253.

30. Ahmed FU, Mahmood BC, Sharma JD, Hoque SM, Zaman R, Hasan MS. Dengue and Dengue Haemorrhagic Fever in Children During the 2000 Outbreak in Chittagong, Bangladesh. Dengue Bulletin 2001; 25: 33-39.

31. Narayanan M. et al. "Dengue fever epidemic in Chennai- A study of clinical profile and outcome." Indian Pediatr. 2002; 39: 1027-1033.

32. Aggarwal Anju et al. "An epidemic of Dengue Hemorrhagic Fever and Dengue Shock Syndrome in children in Delhi." Indian Pediatr. 1998; 35: 727-732.

33. Quiroz-Moreno R, Mendez GF, Ovando- Rivera KM. Clinical Utility of ultrasound in the identification of dengue hemorrhagi fever. Rev Med inst Mex SeguroSoc 2006 44(3): 243-348.

34. Thulkar S, Sharma S, Srivastava DN, Sharma SK, Berry M, Pandey RM et al. Sonographic findings in grade III dengue hemorrhagic fever in adults. J Clin Ultrasound 2000; 28: 34-37.

35. Setiawan MW, Samsi TK, Pool TN ,Sugianto D, Wulur H. Gallbladder wall thickening in dengue hemorrhagic fever: an ultrasonographic Study. J Cin Ultrasound 1995; 23(6): 357-362. 